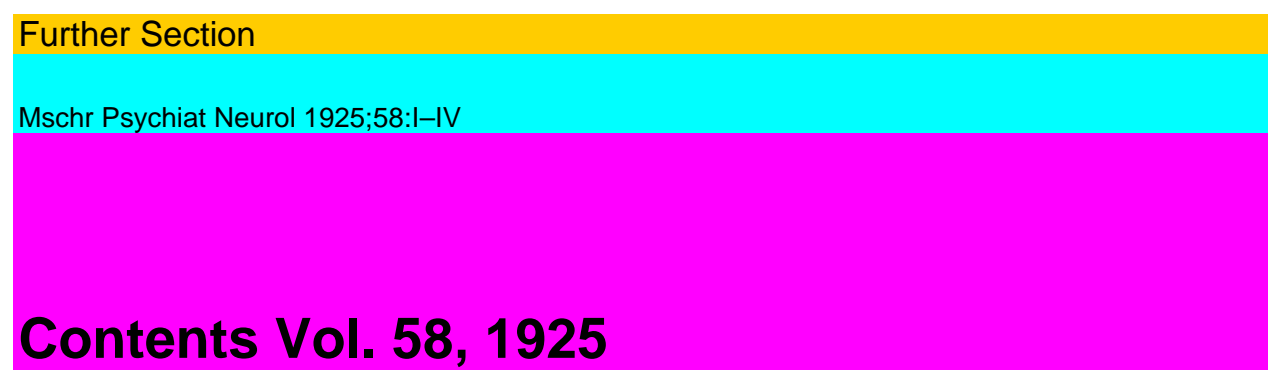

\title{
MONATSSCHIilFT
}

FOR

\section{PSYCHIATRIE UND NEUROLOGIE}

\author{
BEGRUNDET VON C. WERNICKE DND TH. ZIEHEN \\ UNTER MITWIRKUNG VON \\ R. CASSIRER \\ K. KLEIST \\ E. REDLICH \\ FRANKFURT A. M. \\ WIEN \\ P. SCHRODER \\ BERLIN \\ HERAUSGEGEBEN VON \\ K. BOtfHOEFFEB \\ BERLIN
}

Bd. LVIII

Mifc zahlreichen Abbildungen im Text

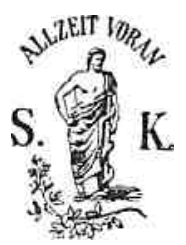

B ERL IN 1925

VERLAG VON S. KARGER

KARLSTRASSE 15. 
Alle Rechte vorbehalten.

Weimar — Druck von li. Wagner Solin. 


\section{Inhalts -Verzeichnis.}

Originalarbeiten.

Seite

Ayala, G., Die Physiopathologie der Mechanik des Li quor cerebrospinalis und der Rachidialquotient 65 Balduzzi, Ottorino, Der Reflex des Malleolus Externus .

Bdlint, A., Sprachiterationen und Psychose bei Ence phalitis epidemica 102

Bohmig, Wolfgang, Statistische Bemerkungen zur Klinik der multiplen Sklerose.

Buckley, Paul, Uber das Verhalten des Blutbilirubins bei Geistes- und Nervenkranken

141

Bychowski, Gustav, Zur Wirkung grofter Cocaingaben

«auf Schizophrene.....

de Crinis, Max, Uber die Beeinflussung des histologi-schen Bildes des Zentralnervensystems durch

humorale Veranderungen 185

Frank, S., Praktische Erfahrungen rait Kastrationen und Sterilisationen psychisch Defekter in der Schweiz.

(Fortsetzung und Schlufi.). 42,148

Gro/Skopf, Siegfr., Ein Fall von traumatischer Hamato-myelie des*9istalen Riickenmarksabschnittes unter besonderer Berucksichtigung seiner Atiologie und Wiirdigung als Unfallerkrankung... .244

Hoff, Hans und Paul Schilder, Uber Lagebeharrung 257

Iwanow-Smolensky, A. G., Uber die bedingten Reflexe in der depressiven Phase des manisch-depressiven

Irreseins

Medow, W., Atypische Psychosen bei Oligophrenie 222, 289 Mutter, $H$. H., Liquorbefund bei multipler Sklerose . . 127 Oseretzky, N., Die motorische Begabung und der Korper-

bau.....

Pineas, Herm., „Palipraxie" oder Nachbewegung? (Mit

Bemerkungen liber „,klonische Perseveration [Lie-p-

mann]) 
Riese, Walter, Zur Psychopathologie des Negativismus

Rosenfeld, M., Uber die Wirkung des Scopolamins auf

die niotorischen Systeme des Zentralnervensystems

1

Schilder, Paul, siehe Hoff, Hans.

Schneider, Carl, । Beitrage zur Lehre von der Schizo

phrenic 5. Mitteilung: Die Bewegungs- und Hand-

lungsstorungen Schizophrener

Straufi, Hans, Uber Sensibilitatsstorungen an Hand und

Gesicht, Geschmacksstorungen und ihre lokalisa-

torische Bedeutung.....

265

Zucker, Konrad, Uber die Wirkung des Physostigmins bei

Erkrankungen des extrapyramidalen Systems . . 11

BuehbespFechungen.....................................................64, 324

Tagesnaehpiehten .................................................................328



